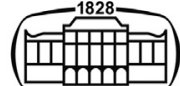

AKADÉMIAI KIADÓ

\section{Acta Veterinaria Hungarica}

$68(2020) 4,387-392$

DOl:

$10.1556 / 004.2020 .00055$

(c) 2021 The Author(s)

\title{
Investigation of the environmental presence of multidrug-resistant bacteria at small animal hospitals in Hungary
}

\section{ÁDÁM KEREK ${ }^{1}$, ÁGNES STERCZER ${ }^{2}$, ZOLTÁN SOMOGYI ${ }^{1}$, DÓRA KOVÁCS ${ }^{1 *} \odot$ and ÁKOS JERZSELE ${ }^{1}$}

\author{
${ }^{1}$ Department of Pharmacology and Toxicology, University of Veterinary Medicine, István u. 2, \\ H-1078 Budapest, Hungary \\ ${ }^{2}$ Department and Clinic of Internal Medicine, University of Veterinary Medicine, István u. 2, H-1078 \\ Budapest, Hungary
}

Received: June 22, 2020 • Accepted: November 5, 2020

Published online: January 5, 2021

\section{ORIGINAL RESEARCH}

\section{PAPER}

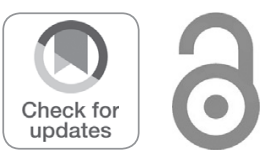

\begin{abstract}
Multidrug-resistant bacteria can cause severe nosocomial infections in both human and veterinary clinics. The aim of this study was to investigate the presence and antibiotic susceptibility of Enterococcus, Staphylococcus and Pseudomonas strains at four small animal clinics of Hungary in 2018, as these bacteria can reliably represent the level of antimicrobial resistance in the investigated environment. A total of 177 Staphylococcus colonies were found, including 22 Staphylococcus pseudintermedius and 13 Staphylococcus aureus. As regards enterococci, 9 Enterococcus faecium, 2 E. faecalis and further 286 Enterococcus strains were isolated. The number of Pseudomonas aeruginosa isolates $(n=34)$ was considered too low for relevant susceptibility testing. Among staphylococci, the highest resistance was found to sulphamethoxazole $(82.9 \%)$, penicillin $(65.7 \%)$ and erythromycin $(54.3 \%)$, while in the case of enterococci, resistance to norfloxacin and rifampicin was the most common, with $25.5 \%$ of the strains being resistant to both antibiotics. Ten methicillin-resistant $S$. pseudintermedius (MRSP) and six vancomycin-resistant Enterococcus (VRE) strains could be identified. Only 5.7\% of the Staphylococcus isolates were susceptible to all tested agents, while this ratio was $36.2 \%$ among enterococci. The results of this study have revealed a high prevalence of antibiotic-resistant bacteria in Hungarian small animal clinics, which highlights the importance of regular disinfection processes and stringent hygiene measures in veterinary clinics.
\end{abstract}

\section{KEYWORDS}

antimicrobial resistance, Staphylococcus, Enterococcus, Pseudomonas, MRSP, VRE

\section{INTRODUCTION}

Nosocomial infections, also known as hospital-acquired or healthcare-associated infections (HAIs), are considered a major threat in human medicine, causing thousands of deaths every year (Suthar et al., 2014; Traverse and Aceto, 2015; Bortolami et al., 2017). The incidence of HAIs is not so well documented in veterinary medicine; however, awareness of their importance is increasing (Traverse and Aceto, 2015; Oh et al., 2018). The bacteria most commonly associated with nosocomial infections include Enterococcus spp., Staphylococcus spp., Salmonella spp., Pseudomonas spp. and Escherichia coli (Mount et al., 2016; Oh et al., 2018; Duarte et al., 2019; Talebi Bezmin Abadi et al., 2019). Acinetobacter baumannii, one of the most clinically significant species in human medicine, has recently been identified as a veterinary nosocomial pathogen (van der Kolk et al., 2019). Multidrug-resistant (MDR) bacteria are especially important in HAIs (Oh et al., 2018). Most of the above-mentioned pathogens belong to the so-called ESKAPE organisms (Enterococcus faecium, Staphylococcus aureus, Klebsiella pneumoniae, A. baumannii, Pseudomonas aeruginosa and Enterobacter
${ }^{*}$ Corresponding author. Tel.: +36 (1) 4784100.

E-mail: kovacs.dora@univet.hu 
species), a group of bacteria with a high rate of antimicrobial resistance that poses a considerable threat in human and veterinary hospitals (van der Kolk et al., 2019).

Besides proper personal hygiene as well as equipment and environmental cleaning and disinfection, the active surveillance of pathogens in small animal clinics is a pivotal step in managing veterinary HAIs (Mount et al., 2016). Numerous international studies have investigated the prevalence of bacteria and their antibiotic susceptibility in veterinary clinics. During a five-year survey (2010-2015) conducted in France, horses, dogs and cats were tested for methicillin-resistant $S$. aureus (MRSA) strains. Of the 130 cases, $60 \%$ were found to be resistant to tetracyclines, $50 \%$ to gentamicin, $48.5 \%$ to enrofloxacin, $33.1 \%$ to erythromycin, and $20.8 \%$ to chloramphenicol (Haenni et al., 2017).

In a 2014 survey, samples were taken from dogs and cats for the examination of Enterococcus species. A total of 115 cases of resistance were identified. PCR identification of the isolates revealed $42(36.5 \%)$ E. faecium and 36 (31.3\%) E. faecalis strains. Thirty-two (27.8\%) bacterial strains were found to be resistant to 2-4 antibiotics, 68 (59.1\%) to 5-9 antibiotics and $15(13 \%)$ to more than 9 antibiotics. The highest resistance (97.5\%) was found against tetracycline (Iseppi et al., 2015).

In a 2017 study in Japan, 200 P. aeruginosa strains were collected from dogs and cats in a veterinary hospital for resistance testing. The study found that $35.5 \%$ of the strains were resistant to phosphomycin, $12.5 \%$ to cefotaxime, $9 \%$ to ciprofloxacin, $4.5 \%$ to gentamicin, and $2.5 \%$ to amikacin (Yukawa et al., 2017).

Environmental sampling performed in an animal hospital of Melbourne has revealed several important sites as possible reservoirs of MDR bacteria. In laundry trolley samples, extended-spectrum beta-lactamase- (ESBL-) producing Klebsiella spp. were found. MDR Pseudomonas strains carrying ampicillin, gentamicin and enrofloxacin resistance genes could be isolated from mop buckets. Intensive care unit cage sampling indicated the presence of penicillin- and quinolone-resistant staphylococci (Kamathewatta et al., 2019).

It should be noted that hospital workers can also contribute to the dissemination of resistant bacteria in veterinary clinics. In a South Korean survey, MDR rate in coagulase-negative staphylococcus isolates from veterinarians was $57.5 \%$, while MDR rate was found to be $44 \%$ in Enterobacteriaceae and only 5\% in Enterococcus spp. (Jung et al., 2020).
Although several international surveys can be found on this subject, the relevant Hungarian literature is rather scarce. Therefore, the aim of this study was to investigate the prevalence and antibiotic susceptibility of $S$. aureus, $S$. pseudintermedius, E. faecalis, E. faecium and P. aeruginosa strains in small animal clinics of Hungary, as these bacteria are highly significant in terms of multidrug resistance and can reliably represent the level of resistance in the investigated environment.

\section{MATERIALS AND METHODS}

\section{Sampling points}

Four small animal clinics were included in this study, some of which work as referral hospitals, dealing with complicated cases on a day-to-day basis. Samples were taken from several critical points, which were chosen based on the estimated risk of possible bacterial contamination. Samples were taken from environmental points where the probability of bacterial presence was assumed to be significant and from hospital workers who can contribute to the maintenance and dissemination of both environmental pathogens and bacteria from hospitalised animals. Samples were taken after regular cleaning processes of the hospitals. The sampling points and the number of samples from each point were similar for all clinics (Table 1). Human samples were taken on a voluntary basis, after receiving verbal consent from the hospital workers involved. Sampling, culturing and susceptibility testing were performed in 2018.

\section{Sampling method}

A transport kit was used for sampling with an Amies type carbonless normal aluminium rod sampler (Copan Italia Spa, Brescia, Italy).

\section{Isolation and differentiation of bacteria}

For the isolation of bacteria, special CHROMagar ${ }^{\mathrm{TM}}$ media (Becton Dickinson GmbH, Germany) were used (different types for staphylococci, enterococci and for P. aeruginosa). Samples were inoculated on the three different media within 1-2 h after collection. From each Petri dish, one colony was used for further studies except for dishes containing extremely high amounts of bacteria. In those cases, two samples were taken from separate parts of the plate with the highest possible distance between them. The differentiation

Table 1. Sampling points and sample numbers

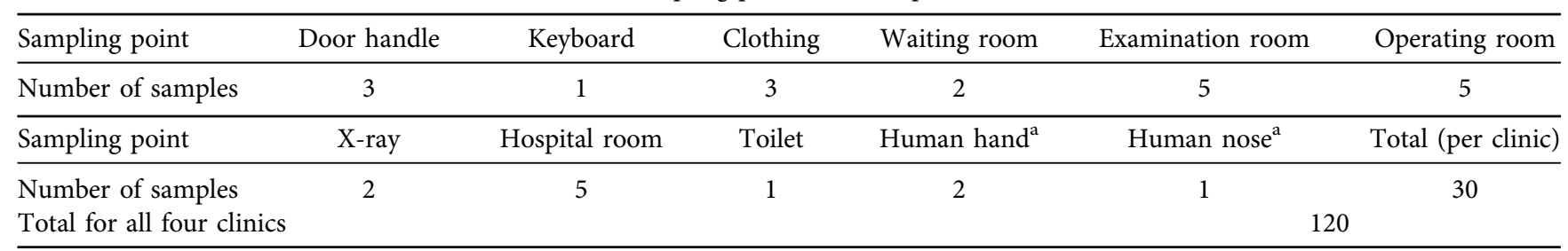

${ }^{\mathrm{a}}$ Voluntary sampling. 
of S. aureus and S. pseudintermedius was performed using pyrrolidonyl aminopeptidase (PYR) assay and PYR reagent (Diagnostics s.r.s., Hodská, Slovakia). For the differentiation of E. faecium and E. faecalis, Enterococcus 8 (ENC 8) tests and alkaline phosphatase (PHS reagent; Diagnostics s.r.s., Hodská, Slovakia) were used.

\section{Disk diffusion test}

Susceptibility testing with the disk diffusion method was based on the CLSI (Clinical \& Laboratory Standards Institute) Performance Standards for Antimicrobial Susceptibility Testing Guideline (Vet08, 4th edition) [Clinical and Laboratory Standards Institute (CLSI), 2018]. After species identification, samples were cultured on tryptone soy agar for 24 $\mathrm{h}$ at $37^{\circ} \mathrm{C}$. Suspensions of each bacterial strain were prepared in sterile saline $(0.85 \% \mathrm{NaCl})$ to obtain a suspension of 0.5 McFarland $\left(1-2 \times 10^{8}\right.$ suspended bacteria/ml) and then inoculated into two Petri dishes. Following inoculation, antibiotic disks were applied on the bacteria. Each Petri dish was treated with 10 different types of antibiotics (Abtek Biologicals Ltd, Liverpool, United Kingdom), chosen on the basis of the common susceptibility profile of the species.

\section{Determination of Minimum Inhibitory Concentration (MIC) value}

Against staphylococci, vancomycin and oxacillin do not give reliable results with the disk diffusion method, therefore the MIC values of these antibiotics were determined. Serial dilutions from $16 \mathrm{mg} / \mathrm{L}$ to $0.03125 \mathrm{mg} / \mathrm{L}$ for oxacillin and from $64 \mathrm{mg} / \mathrm{L}$ to $0.125 \mathrm{mg} / \mathrm{L}$ for vancomycin were prepared.

\section{RESULTS}

The number of pathogens at each site is shown in Fig. 1. Among the four clinics, significant amount of enterococci grew out from samples taken from computer keyboards at site IV (18 colonies) and from the floor of the hospital cages at site II (50 colonies), while this was not observed at other clinics. Further Enterococcus strains could be isolated from surgical gowns at sites I and II (9 and 4 colonies, respectively). As regards staphylococci, 50 strains each grew out from cages and nasal samples taken at clinic I, while at site II 50 colonies were isolated from muzzles. P. aeruginosa (30 isolates) could only be cultured from washbasins at site III. A significant number of $E$. coli strains was also isolated from keyboards, muzzles, and surgical gowns.

From the obtained bacteria, we performed further tests on a limited number of colonies only, namely one per site and per bacterium species.

Thirty-five out of the 177 Staphylococcus (mauve) colonies were tested with the PYR test. Based on the test results, 22 samples were PYR positive ( $S$. pseudintermedius), while 13 samples were PYR negative (S. aureus).

The ENC 8 test was performed on 96 samples from 297 colonies of Enterococcus (turquoise blue). We found 9 E. faecium and 2 E. faecalis strains.

Only three different colonies of the $34 \mathrm{P}$. aeruginosa isolates were tested further. The number of $P$. aeruginosa isolates was too low to provide relevant percentage results regarding their antibiotic susceptibility.

For Staphylococcus species, the highest resistance was observed to sulphamethoxazole, with $82.9 \%$ of the strains being resistant. Furthermore, $65.7 \%$ of the isolates were resistant to penicillin and $54.3 \%$ to erythromycin. Overall, $28.6 \%$ of the strains were found to be resistant to oxacillin (by MIC determination), which included all strains of $S$. pseudintermedius. Of the Staphylococcus strains, $22.9 \%$ were resistant to gentamicin, $20 \%$ to chloramphenicol, $11.4 \%$ to rifampicin, $5.7 \%$ to ciprofloxacin and $5.7 \%$ to doxycycline. Vancomycin resistance was not found in staphylococci (Table 2). A species-identified S. aureus subsp. aureus strain isolated from a veterinarian's nasal mucosa was found to be resistant to 7 antibiotics (sulphamethoxazole, penicillin,

Colony count of pathogens at each site (pcs)

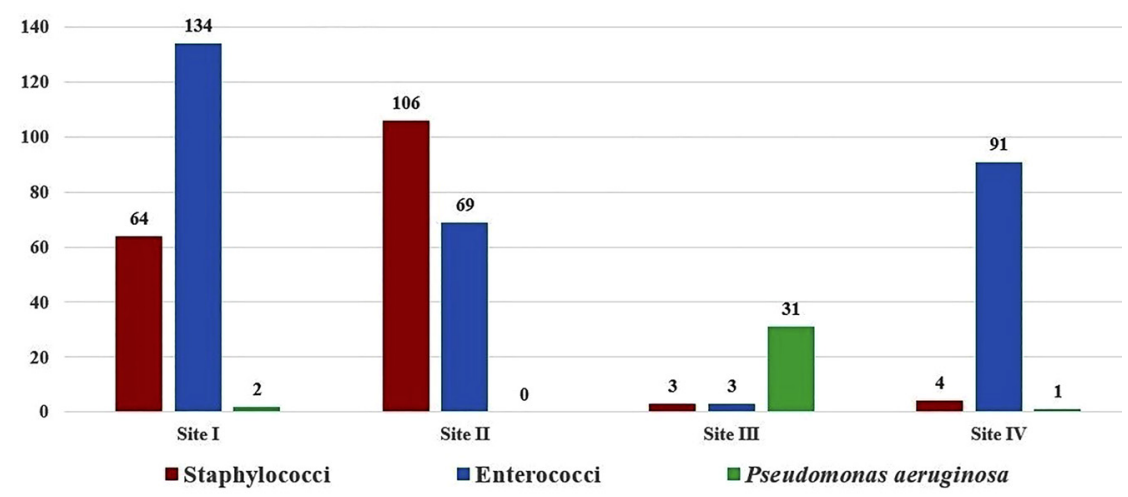

Fig. 1. The figure shows the colony counts of pathogens isolated from 120 samples collected at four small animal clinics. The Staphylococcus count was highest at Site II $(n=106)$, while the highest number of enterococci was found at Site I $(n=134)$. The highest amount of staphylococci could be isolated from samples obtained from hospital floor and muzzles. Most of the Enterococcus colonies grew out from samples of the nasal mucosa, cage grids and computer keyboards 


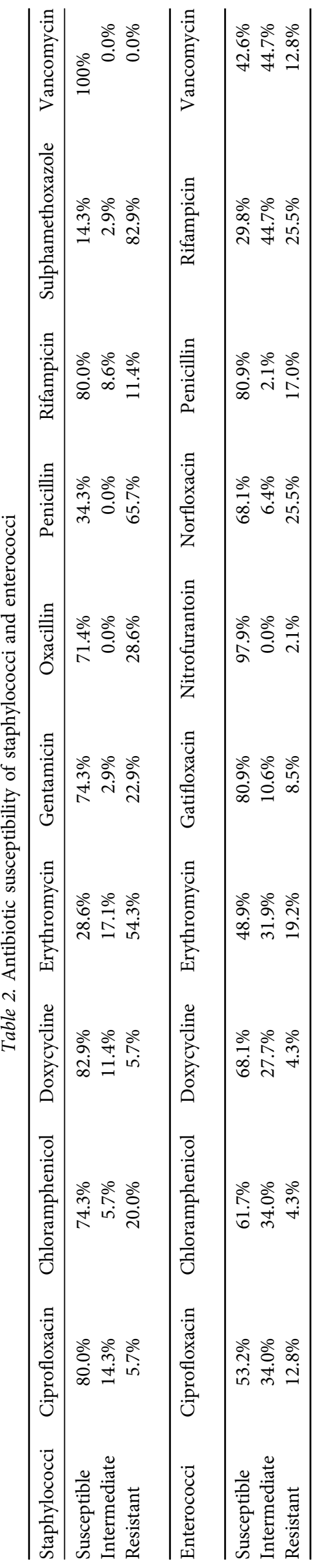

erythromycin, gentamicin, ciprofloxacin, doxycycline, and chloramphenicol). No MRSA strains were found; however, 10 methicillin-resistant S. pseudintermedius (MRSP) strains could be identified. Overall, $85.7 \%$ of the isolated Staphylococcus strains were multidrug resistant, $8.6 \%$ were resistant to only one antibiotic and $5.7 \%$ were fully susceptible to all agents tested.

In the current investigation, Enterococcus isolates showed the highest resistance to rifampicin and norfloxacin, with $25.5 \%$ of the strains being resistant to each of the two agents. The resistance rates of Enterococcus strains to other antibiotics were as follows: $19.2 \%$ of the strains were resistant to erythromycin, $17 \%$ to penicillin, $12.8 \%$ to vancomycin, $12.8 \%$ to ciprofloxacin, $8.5 \%$ to gatifloxacin, $4.3 \%$ to doxycycline, and $4.3 \%$ to chloramphenicol. The lowest resistance was observed against nitrofurantoin, with $2.1 \%$ of the isolates being resistant (Table 2). Six vancomycin-resistant Enterococcus (VRE) strains were found. Two strains were found to be resistant to 7 antibiotics, one of them was isolated from a computer keyboard, while the other from the floor of a hospital. Overall, 23.4\% of the isolated Enterococcus strains were multidrug resistant, $40.4 \%$ of them were resistant to only one antibiotic, and $36.2 \%$ were fully susceptible to all agents tested.

Among the P. aeruginosa isolates tested, one strain was resistant to aztreonam, which was isolated from the floor of a hospital. Another strain, isolated from a sink, was multidrug resistant (aztreonam, gentamicin, tobramycin and ciprofloxacin resistance), while one pan-resistant (imipenem, ofloxacin, tobramycin and polymyxin B resistance) (PDR) strain was found on a surgical gown. Interpretation of these results as percentages was not considered relevant due to the low number (3) of $P$. aeruginosa strains tested.

\section{DISCUSSION}

In this study, a total of 120 samples were taken from critical points of four small animal clinics for bacterial culture and antimicrobial susceptibility testing. Antimicrobial susceptibility of the bacteria was tested by the disk diffusion method, except for the susceptibility of staphylococci to oxacillin and vancomycin. For these, MIC value determination was performed.

The most important predilection sites for staphylococci included muzzles, surgical gowns, and floor of the hospital cages. For enterococci, predilection sites were partially similar, including computer keyboards, surgical gowns and floor of the hospital cages.

The number of Staphylococcus and Enterococcus samples ( $n=177$ and $n=297$, respectively) was sufficient for a percentage-based evaluation of data. These might have contained colonies of the same lineage, but even bacterial samples taken from the same agar showed different resistance profiles. The number of $P$. aeruginosa colonies $(n=$ 34) was not sufficient to calculate resistance rates; however, it should be noted that both MDR and PDR P. aeruginosa strains were found during this investigation. 
In harmony with the findings of the current study, methicillin-resistant staphylococci can frequently be isolated in veterinary clinics. In the work of Fessler et al. (2018), samples were taken from the environment and employees of a small animal hospital in Germany. The authors found 11 MRSA and 7 MRSP strains, which displayed additional resistance properties to fluoroquinolones, macrolides and lincosamides. Oh et al. (2018) conducted a survey in a Korean veterinary hospital, where a total of 9 methicillinresistant staphylococci could be identified, 4 of which were considered multidrug resistant, showing resistance to azithromycin, oxacillin, penicillin, clindamycin, and gentamicin. Fungwithaya et al. (2017) examined samples collected in a veterinary hospital of Bangkok. They found that in the operating room, all rebreathing circuits were positive for staphylococci, including MRSP; furthermore, MRSP, MRSA and MSSP (methicillin-sensitive S. pseudintermedius) strains could be isolated from the incision sites of patients who had gone through standard aseptic preparation. In contrast to the current and the above-mentioned studies, Worthing et al. (2018) did not find any MRSP or MRSA strains in the environment of two small animal hospitals in Australia, but they could isolate MRSA from veterinary personnel. Although the current study only focused on small animal clinics, it should be noted that the prevalence of MRSA strains is frequently revealed in equine clinics as well (Bortolami et al., 2017; van Spijk et al., 2017). Furthermore, not only veterinary clinics can serve as potential sources of MDR bacteria of animal origin. Trościańczyk et al. (2020) investigated the antibiotic susceptibility profile of staphylococci isolated from canine and feline faecal samples collected from urban spaces. They have also found $14 \%$ of the isolates to be multidrug resistant, with high resistance rates to tetracycline (60\%) and penicillin (58\%).

Overall, these rates of antibiotic resistance among enterococci can be considered low compared to the results of Chung et al. (2014), who conducted a survey in five veterinary hospitals in Korea. They found very high rates of resistance in E. faecalis and E. faecium strains against several antibiotics, e.g. $81.3 \%$ to tetracycline and ampicillin, $68.8 \%$ to erythromycin and ciprofloxacin, $56.3 \%$ to gentamicin, and $37.5 \%$ to streptomycin. The prevalence of multidrug-resistant enterococci was also higher among their isolates, reaching $75 \%$ of E. faecium and $62.5 \%$ of E. faecalis strains. However, in contrast to the current study, no VRE strains could be detected by Chung et al. (2014). KuKanich et al. (2012) investigated Enterococcus strains in ten small animal veterinary hospitals in Kansas. According to their results, high proportions of E. faecium strains were resistant to several antibiotics [e.g. enrofloxacin (73.0\%), erythromycin (53.9\%), ampicillin (51.0\%) and doxycycline (42.9\%)], while E. faecalis isolates were more susceptible to the agents tested. Like in the work of Chung et al. (2014) and in contrast with the current study, KuKanich et al. (2012) could not detect resistance to vancomycin. The first VRE isolated from a worker of a small animal hospital was reported by Oh et al. (2018) after a Korean survey in 2014.
As a conclusion of the present investigation, it can be stated that the prevalence of antibiotic-resistant bacterial strains in Hungarian small animal clinics is abundant. Even though some of the isolated bacteria might not be pathologically relevant, their resistance profile can serve as an indicator of the resistance status of the clinics involved. Our findings underline the importance of regular disinfection processes and stringent hygiene measures at small animal clinics. Discrepancies in the levels of resistance between the current study and earlier surveys may be due to differences in the date and location of the surveys and, in connection with the latter, the diverse antibiotic usage of the countries involved.

\section{ACKNOWLEDGEMENTS}

The research was supported by the New National Excellence Program, award number UNKP-17-2-I-TAB-2 of the Ministry of Human Resources.

\section{REFERENCES}

Bortolami, A., Williams, N. J., McGowan, C. M., Kelly, P. G., Archer, D. C., Corrò, M., Pinchbeck, G., Saunders, C. J. and Timofte, D. (2017): Environmental surveillance identifies multiple introductions of MRSA CC398 in an equine veterinary hospital in the UK, 2011-2016. Sci. Rep. 7, 5499.

Chung, Y. S., Kwon, K. H., Shin, S., Kim, J. H., Park, Y. H. and Yoon, J. W. (2014): Characterization of veterinary hospitalassociated isolates of Enterococcus species in Korea. J. Microbiol. Biotechnol. 24, 386-393.

Clinical and Laboratory Standards Institute (CLSI) (2018): Performance Standards for Antimicrobial Disk and Dilution Susceptibility Tests for Bacteria Isolated from Animals. CLSI document Vet08, 4th ed. Clinical and Laboratory Standards Institute, Wayne, PA.

Duarte, B., Pereira, A. P., Freitas, A. R., Coque, T. M., Hammerum, A. M., Hasman, H., Antunes, P., Peixe, L. and Novais, C. (2019): 2CS-CHXT operon signature of chlorhexidine tolerance among Enterococcus faecium isolates. Appl. Environ. Microbiol. 85 (23), e01589-e01519.

Fessler, A. T., Schuenemann, R., Kadlec, K., Hensel, V., Brombach, J., Murugaiyan, J., Oechtering, G., Burgener, I. A. and Schwarz, S. (2018): Methicillin-resistant Staphylococcus aureus (MRSA) and methicillin-resistant Staphylococcus pseudintermedius (MRSP) among employees and in the environment of a small animal hospital. Vet. Microbiol. 221, 153-158.

Fungwithaya, P., Brikshavana, P., Chanchaithong, P. and Prapasarakul, N. (2017): Distribution of methicillin-resistant coagulase-positive staphylococci (MRCoPS) in a surgical unit and cystotomy operation sites in a veterinary teaching hospital. J. Vet. Med. Sci. 79, 359-365.

Haenni, M., Châtre, P., Dupieux-Chabert, C., Métayer, V., Bes, M., Madec, J-Y. and Laurent, F. (2017): Molecular 
epidemiology of methicillin-resistant Staphylococcus aureus in horses, cats, and dogs over a 5 -year period in France. Front. Microbiol. 8, 2493.

Iseppi, R., Messi, P., Anacarso, I., Bondi, M., Sabia, C., Condó, C. and de Niederhausern, S. (2015): Antimicrobial resistance and virulence traits in Enterococcus strains isolated from dogs and cats. New Microbiol. 38, 369-378.

Jung, W. K., Shin, S., Park, Y. K., Lim, S-K., Moon, D-C., Park, K. T. and Park, Y. H. (2020): Distribution and antimicrobial resistance profiles of bacterial species in stray cats, hospitaladmitted cats, and veterinary staff in South Korea. BMC Vet. Res. 16, 109.

Kamathewatta, K. I., Bushell, R. N., Young, N. D., Stevenson, M. A., Billman-Jacobe, H., Browning, G. F. and Marenda, M. S. (2019): Exploration of antibiotic resistance risks in a veterinary teaching hospital with Oxford Nanopore long read sequencing. PloS One 14 (5), e0217600.

KuKanich, K. S., Ghosh, A., Skarbek, J. V., Lothamer, K. M. and Zurek, L. (2012): Surveillance of bacterial contamination in small animal veterinary hospitals with special focus on antimicrobial resistance and virulence traits of enterococci. J. Am. Vet. Med. Assoc. 240, 437-445.

Mount, R., Schick, A., Lewis, T. and Newton, H. (2016): Evaluation of bacterial contamination of clipper blades in small animal private practice. J. Am. Anim. Hosp. Assoc. 52, 95-101.

Oh, Y-I., Baek, J. Y., Kim, S. H., Kang, B-J. and Youn, H-Y. (2018): Antimicrobial susceptibility and distribution of multidrugresistant organisms isolated from environmental surfaces and hands of healthcare workers in a small animal hospital. Japan. J. Vet. Res. 66, 193-202.
Suthar, N., Roy, S., Call, D., Besser, T. and Davis, M. (2014): An individual-based model of transmission of resistant bacteria in a veterinary teaching hospital. PloS One 9 (6), e98589.

Talebi Bezmin Abadi, A., Rizvanov, A., Haertlé, T. and Blatt, N. (2019): World Health Organization report: current crisis of antibiotic resistance. BioNanoSci. 9, 778-788.

Traverse, M. and Aceto, H. (2015): Environmental cleaning and disinfection. Vet. Clin. North Am. Small Anim. Pract. 45, 299330.

Trościańczyk, A., Nowakiewicz, A., Gnat, S., Wójcik, M., WdowiakWróbel, S. and Kalita, M. (2020): Contamination of the urban environment with excrements of companion animals as an underestimated source of Staphylococcus species posing a threat to public health. Acta Vet. Hung. 68, 12-19.

van der Kolk, J., Endimiani, A., Graubner, C., Gerber, V. and Perreten, V. (2019): Acinetobacter in veterinary medicine, with an emphasis on Acinetobacter baumannii. J. Glob. Antimicrob. Resist. 16, 59-71.

van Spijk, J. N., Schmitt, S. and Schoster, A. (2017): Infections caused by multidrug-resistant bacteria in an equine hospital (2012-2015). Eq. Vet. Educ. 31, 653-658.

Worthing, K., Brown, J., Gerber, L., Trott, D., Abraham, S. and Norris, J. (2018): Methicillin-resistant staphylococci amongst veterinary personnel, personnel-owned pets, patients and the hospital environment of two small animal veterinary hospitals. Vet. Microbiol. 223, 79-85.

Yukawa, S., Tsuyuki, Y., Sato, T., Fukuda A., Usui M. and Tamura Y. (2017): Antimicrobial resistance of Pseudomonas aeruginosa isolated from dogs and cats in primary veterinary hospitals in Japan. Japan. J. Infect. Dis. 70, 461-463. 